\title{
Retrieval of aerosol optical properties from ground-based remote sensing measurements: Aerosol asymmetry factor and single scattering albedo
}

\author{
L. Qie, ${ }^{1}$, Z. $\mathrm{Li}^{1}{ }^{1} *$, L. $\mathrm{Li}^{1}, \mathrm{~K} . \mathrm{Li}^{1}, \mathrm{D} \cdot \mathrm{Li}^{1}, \mathrm{H} . \mathrm{Xu}{ }^{1}$ \\ ${ }^{1}$ State Environmental Protection Key Laboratory of Satellite Remote Sensing, Institute of Remote Sensing and Digital Earth, \\ Chinese Academy of Sciences, Datun Road, Beijing, China - (qiell, lizq, lili3, likt, lidh, xuhua)@ radi.ac.cn
}

\author{
Commission VI, WG VI/4
}

KEY WORDS: Devaux-Vermeulen-Li method, aerosol, single scattering albedo, asymmetry factor, Sun-sky radiometer, AERONET

\begin{abstract}
:
The Devaux-Vermeulen-Li method (DVL method) is a simple approach to retrieve aerosol optical parameters from the Sun-sky radiance measurements. This study inherited the previous works of retrieving aerosol single scattering albedo (SSA) and scattering phase function, the DVL method was modified to derive aerosol asymmetric factor (g). To assess the algorithm performance at various atmospheric aerosol conditions, retrievals from AERONET observations were implemented, and the results are compared with AERONET official products. The comparison shows that both the DVL SSA and g were well correlated with those of AERONET. The RMSD and the absolute value of MBD deviations between the SSAs are 0.025 and 0.015 respectively, well below the AERONET declared SSA uncertainty of 0.03 for all wavelengths. For asymmetry factor g, the RMSD deviations are smaller than 0.02 and the absolute values of MBDs smaller than 0.01 at 675,870 and $1020 \mathrm{~nm}$ bands. Then, considering several factors probably affecting retrieval quality (i.e. the aerosol optical depth (AOD), the solar zenith angle, and the sky residual error, sphericity proportion and Ángström exponent), the deviations for SSA and g of these two algorithms were calculated at varying value intervals. Both the SSA and $g$ deviations were found decrease with the AOD and the solar zenith angle, and increase with sky residual error. However, the deviations do not show clear sensitivity to the sphericity proportion and Ángström exponent. This indicated that the DVL algorithm is available for both large, non-spherical particles and spherical particles. The DVL results are suitable for the evaluation of aerosol direct radiative effects of different aerosol types.
\end{abstract}

\section{INTRODUCTION}

Accurate knowledge of aerosol spatial and temporal properties is of great importance for research of climate forcing, atmospheric environment etc. Ground-based Sun-sky radiometer, in principle, is one of the most accurate and simplest aerosol remote sensing systems. There have been numerous important ground-based aerosol observation networks were developed, for example, AERONET (Holben et al., 1998), SKYNET (Nakajima et al., 1996) and SONET (Sun-sky Radiometer Observation Network, Li et al., 2015). Sun-sky radiometer measures the spectral direct Sun radiation and angular sky radiance and polarization at atmospheric window bands. The direct solar radiation measurements provide accurate total aerosol optical depth (AOD), and the sky radiances are used to derive aerosol microphysical (such as size distribution, complex reflective index) and optical properties (such as single scattering albedo (SSA), scattering phase function, asymmetry factor (g)) through elaborate inversion algorithms. These networks provide reliable global or regional aerosol properties products via imposing standardization instruments, unified strict quality controls, data processing procedures and inversion algorithm, and these data has been extensively used in the studies of aerosol characteristics, typical aerosol modelling, satellite retrievals validation and aerosol effects on climate forcing.

Aerosols influence the Earth energy budget directly by scattering and absorbing the incoming solar radiation. SSA and g are two of the key parameters that determine the aerosols direct radiative effects. SSA is defined as the ratio between the aerosol scattering and extinction coefficients, it describes aerosol absorption. The magnitude and sign of aerosol radiative forcing are determined, to a large extent, by the value and of SSA (Hansen et al. 1997).
The $g$ is defined as the first moment of the scattering phase function, it denotes the relative strength of forward scattering, which determines the integrated fractions of energy backscattered and forward scattered. For aerosol particles, whose phase function has a generally sharp peak at $0^{\circ}$ scattering angle, $\mathrm{g}$ is positive, and increases with the particle size. Accurate measurements of SSA and $\mathrm{g}$ are critical for the modeling estimates of aerosol climate forcing (Yu et al. 2006).

There have been continuous efforts devoted to developing inversion methods for deriving aerosol optical and/or physical properties from ground-based measurements. The two of most important operational algorithms are these for Skynet and AERONET, which are developed by Nakajima et al. (1996) and Dubovik et al. (2000), respectively. The Devaux-Vermeulen-Li method (DVL method, Devaux et al., 1998; Vermeulen et al., 2000; Li et al., 2006) is another approach to retrieve aerosol SSA and phase function based on combined measurements of solar transmission and scanning of sky radiance. The theoretical scheme of this approach is that, the solar transmission provide AOD for extinction, while the scanning of sky radiance provide angular distribution of aerosol scattering phase function. Base on the normalization of the aerosol phase function, the AOD for scattering can be deduced, and then providing the SSA by AODsca/AODext. Compared to the algorithms of Skynet and AERONET, the DVL method requires no assumptions with respect to the aerosol microphysical properties, and it is much simpler, more comprehensible and easier to implement compared to the complex statistical optimization theory and inversion strategies.

This study inherits previous work of DVL retrieving aerosol SSA and scattering phase function, and make further efforts to

* Corresponding author 
calculate the asymmetry factor g. The algorithm are applied to AERONET Beijing site measurements, and the SSA and $g$ retrievals were systematically compared with AERONET version 2 level 2.0 products, and the factors probably affecting the retrievals quality are investigated.

\section{DVL METHOD DESCRIPTION}

We introduce the DVL method below very briefly. The kernel of this approach is to eliminate the contributions of ground reflection, Rayleigh scattering and multiple scatterings from the sky radiance measurements to retrieve the single-scattering properties of atmospheric aerosols. As long as the estimated aerosol model are close to the actual measurements, the (single scattering)/(total scattering) ratio is expected to be approximately equal for simulations and measurements of sky radiance $(L)$. Then the relationship between the actual and estimated (marked with a superscript $*$ ) sky radiance can be obtained as,

$$
\frac{\omega_{0} p_{a}}{\omega_{0}^{*}}=\frac{L-\Delta L^{*}}{L(0)^{*}} p_{a}^{*}+\frac{L-L^{*}}{L(0)^{*}} \frac{\tau_{m}}{\omega_{0}^{*} \tau_{a}} p_{m}(1)
$$

$\Delta L \approx \Delta L^{*}=L^{*}\left(\rho_{g}^{*}\right)-L^{*}(0)$ is approximately the surface contribution. $\tau_{m}$ and $p_{m}$ are the molecular optical depth and phase function, respectively. $\tau_{a}$ is AOD that can be measured accurately. Normalization of the aerosol phase function yields,

$$
\int_{0}^{\pi} p_{a}(\theta) \sin (\theta) d \theta=2(2)
$$

where $\theta$ is the scattering angle. Then, Eq. (1) leads to

$$
\frac{2 \omega_{0}}{\omega_{0}^{*}}=\int_{0}^{\pi}\left(\frac{L-\Delta L^{*}}{L(0)^{*}} p_{a}^{*}+\frac{L-L^{*}}{L(0)^{*}} \frac{\tau_{m}}{\omega_{0}^{*} \tau_{a}} p_{m}\right) \sin (\theta) d \theta(3)
$$

Obviously, with the right-hand integral equal to 2 , it leads to $\omega_{0}^{*}=\omega_{0}$ on the left-hand side, i.e., the real SSA value is retrieved. Substituting the retrieved $\omega_{0}$ into Eq. (1), the aerosol phase function can be retrieved.

The asymmetry factor of the phase function is defined as

$$
g=\frac{1}{2} \int_{0}^{\pi} p(\theta) \cos (\theta) \sin (\theta) d \theta(4)
$$

By replacing the retrieved aerosol phase function $p_{a}(\theta)$ in Eq. (4), $\mathrm{g}$ is derived by calculating the integral over the entire scattering angle of $0^{\circ}-180^{\circ}$. For the Sun-sky radiometer's almucantar (ALM) observation, the maximum observed scattering angle $\theta_{\max }$ equals $2 \theta_{s}, \theta_{s}$ is the solar zenith angle, and the minimum observed scattering angle $\theta_{\min }$ is approximately $3^{\circ}$. Therefore, the forward missing phase function is extrapolated by using the shape of measured phase function near the non-accessible range $\left[\theta_{\min }, \theta_{\min }+3^{\circ}\right]$, by using the quadratic polynomial, and the backscattering angles $\left[\theta_{\max }, 180^{\circ}\right]$ was estimated by the initially estimated phase function $p_{a}^{*}$. Then, $\mathrm{g}$ is calculated according to Eq. (4).

\section{APPLICATION TO AERONET OBSERVATIONS}

\subsection{Site and data description}

To assess the DVL algorithm performance at various atmospheric aerosol conditions, retrievals from AERONET observations were implemented, and the SSA and g results are compared with AERONET official products.

We selected the Beijing site (Lon=116.381, Lat=39.977) observations of AERONET, from January 2011 to March 2015, for this study. As the capital of China, Beijing is one of the most densely populated area. Its complex and highly variable aerosol emission sources and compositions, including local sources of traffic emissions and fossil fuel combustion, and long-range transport of dust and industrial emissions from neighbouring regions, make it convenient for the aerosol sample selection and algorithm evaluation.

\subsection{Retrieval process}

To make the DVL results comparable with the AERONET algorithm, the almucantar sky radiance data and AOD data of the Sun-sky radiometer at Beijing site were selected for the retrieval. Since only the retrieval of ALM observations instead of the PPL observations are included in the level 2.0 AERONET products, considering the results reliability. The sky radiance data had been well calibrated, and the AOD were derived from the direct solar measurement. Additionally, several essential pre-processing procedures for ALM radiance data quality check were performed, referring to the AERONET's Version 2.0 quality assurance criteria (Holben, 2002), including the A-K check to validate the sky radiance calibration, the symmetry check of the left and the right almucantar branches to eliminate cloud contaminated data, the average of the remaining quality assured left and right branches of radiance and also their normalization. The MODIS surface albedos products were utilized as our input surface parameters, following AERONET, too. Then the normalized radiance and AOD, surface albedo data were input into the DVL algorithm, and the aerosol SSA and asymmetry factors were retrieved at 440, 675, 870 and $1020 \mathrm{~nm}$ bands.

\section{COMPARISON WITH AERONET PRODUCTS}

\subsection{Comparison methodology}

The level 2.0 products provided by AERONET are considered quality assured. A series of criteria are required for the data quality control, including the raw data quality of the measurements, the calibration check for the aureole and sky radiance (i.e. A-K criteria), the scattering angle range criteria (depends on the solar zenith angle), and the residual error criteria (residual error between the measured and simulated sky radiance of the retrievals). In addition, SSA of level 2.0 (with accuracy to the level of 0.03$)$ need to satisfy that AOD $(440 \mathrm{~nm}) \geqslant 0.4$. For the SSA with lower aerosol loading, i.e. AOD $(440 \mathrm{~nm})<0.2$, the accuracy level drop down to 0.05-0.07.

The DVL retrievals of SSA and $g$ were compared with AERONET version 2 level 2.0 product. We considered five factors probably affecting retrieval quality, i.e. the aerosol optical depth (AOD), the solar zenith angle, and the sky residual error, sphericity proportion and Ángström exponent, the deviations for SSA and $g$ of these two algorithms were calculated at varying value intervals. We retained only aerosol samples with AOD (440 $\mathrm{nm})>0.4$ for the analysis to guarantee the retrieval reliability except for the factor of AOD, and a total of 1,120 sample pairs were acquired during this period. To analysis the conditions of low AOD, the samples with $0.05<$ AOD440 $<0.4$ were selected form the AERONET level 1.5 data, who satisfied all the other level 2.0 criteria except for the AOD criteria.

To estimate the deviation between DVL SSA and $g$ retrievals and AERONET products, several statistical indicators have been calculated for the comparison: root mean square deviation (RMSD), mean bias deviation (MBD) and standard deviation of differences (STD).

$$
\begin{gathered}
\operatorname{RMSD}=\sqrt{\frac{1}{N} \sum_{i=1}^{N}\left(x_{i}-x_{0 i}\right)^{2}}(5 \mathrm{a}) \\
\operatorname{MBD}=\bar{\Delta}=\frac{1}{N} \sum_{i=1}^{N}\left(x_{i}-x_{0 i}\right)=\frac{1}{N} \sum_{i=1}^{N} \Delta_{i}(5 \mathrm{~b}) \\
\operatorname{STD}=\sqrt{\frac{1}{N} \sum_{i=1}^{N}\left(\Delta_{i}-\bar{\Delta}\right)^{2}}(5 \mathrm{c})
\end{gathered}
$$

Here, $\mathrm{x}$ indicates the quantity of SSA or $\mathrm{g}, x_{0 i}$ and $x_{i}$ refer to the AERONET product and DVL retrieval respectively, $\mathrm{N}$ is the number of samples. 
Figure1 shows the frequency distribution of data samples change with the five factors.

a) AOD $(440 \mathrm{~nm})$

As shown in Figure 1, the AOD $(440 \mathrm{~nm})$ of the samples ranges from $0.05-5.0$. It is divided into 12 intervals. The 1 st interval is $0.05-0.2$. The 2 nd to 10 th intervals is $0.2-2.0$, with an interval of 0.2. The 11th and 12th intervals are $2.0-3.0$ and $3.0-5.0$, respectively. It is noteworthy that, the samples for AOD $(440 \mathrm{~nm})$ $<0.4$ accounted for more than half $(51 \%)$ of the total number of samples. However, only level 1.5 products of AERONET are available.

b) Solar zenith angle

AERONET's level 2.0 products requires $\mathrm{SZ} \geqslant 50^{\circ}$. The solar zenith angle ranges from $50^{\circ}-80^{\circ}$, which was divided into 6 equal intervals of $5^{\circ}$. The samples are relatively balanced distributed in each SZ interval.

c) The sky residual error

AERONET's level 2.0 products requires the sky residual error $\leqslant$ $8 \%$. The sky residual error ranges from $0.0 \%-8.0 \%$, which was divided into 8 equal intervals of $1.0 \%$. As shown, the number of samples with sky residual error $<1.0 \%$ and Sky residual error $>$ $6.0 \%$ are small. The subsequently analysis focuses on the samples with sky residual errors from $1.0 \%-6.0 \%$.

d) The spherical proportion of particles

The spherical proportion is an important indicators for the aerosol particles shape. It ranges from $0.0 \%-100.0 \%$, and has been divided into 10 equal intervals. The distribution of samples are relatively uniform in the intervals from $10.0 \%-80.0 \%$ except for the intervals of $0.0-1.0 \%$ and $98.0 \%-99 \%$. There are respectively 45 and 132 samples distributed in these 2 small intervals, which is related to the processing of the AERONET inversion algorithm.

e) Ángström exponent

The Ångström exponent $(440-870 \mathrm{~nm})$ ranges from $0.0-2.0$. It is divided into 10 equal intervals. The samples are mainly concentrated in the interval of $0.8-1.6$, which accounts for about $89.0 \%$ of the total number of samples.
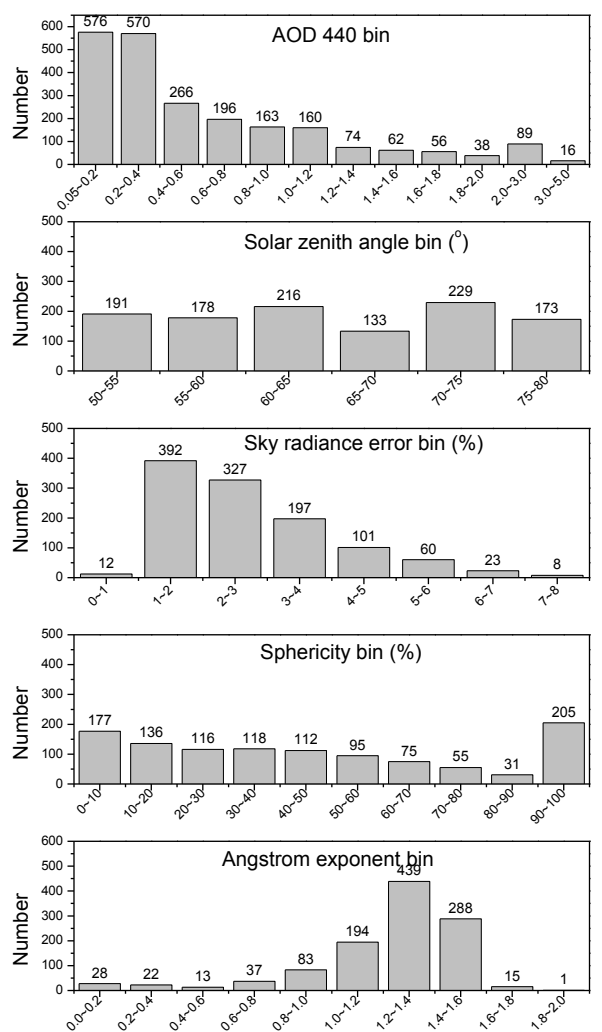

Figure 1 The frequency distribution of data samples

\subsection{SSA comparison}

Figure 2 shows the RMSD and MBD deviations of the SSAs retrieved by DVL and AERONET, as a function of AOD (440 $\mathrm{nm}), \mathrm{SZ}$, Sky error, Sphericity and Ángström exponent, at 4 wavelengths of 440, 675, 870 and $1020 \mathrm{~nm}$, respectively.

a) Change with AOD $(440 \mathrm{~nm})$

In general, the SSAs deviations (RMSD, MBD and STD) of DVL and AERONET decrease the AOD $(440 \mathrm{~nm})$ for the 4 wavelengths. When AOD $(440 \mathrm{~nm})>0.4$, the RMSD and MBD deviations are smaller than 0.03 , and tend to be stable when AOD $(440 \mathrm{~nm})>1.0$, with values of about 0.01 . For the low aerosol loading conditions, i.e. AOD $(440 \mathrm{~nm}) \leqslant 0.4$, the RMSD of the 4 wavelengths increase to $0.03-0.06$. The deviations are within the AERONET SSA error limit of $0.05-0.07$ that evaluated by Dubovik (2000).

b) Change with SZ

The SSAs deviation of RMSD are less than 0.03 in all SZ intervals. The MBDs of the 4 wavelengths decrease as SZ increase.

c) Change with Sky error

For the sky residual errors range of $1.0 \%-6.0 \%$, the SSAs deviations (RMSD, MBD and STD) increase with the Sky error at all 4 wavelengths.

d) Change with spherical particles proportion

The SSAs deviations (RMSD, MBD and STD) do not change significantly with the particles' spherical proportion. The RMSD and absolute value of MBD are less than 0.03 in all sphericity intervals. This indicate that the DVL algorithm retrievals of SSA are not sensitive to the particles shape.

e) Change with the Ángström exponent

It's similar to that of spherical proportion, the SSAs deviations (RMSD, MBD and STD) do not show any obvious trend with the Ángström exponent, and the RMSD and absolute value of MBD are less than 0.03 in all Ángström exponent intervals. This indicate that the DVL algorithm retrievals of SSA are not sensitive to the particles size too.
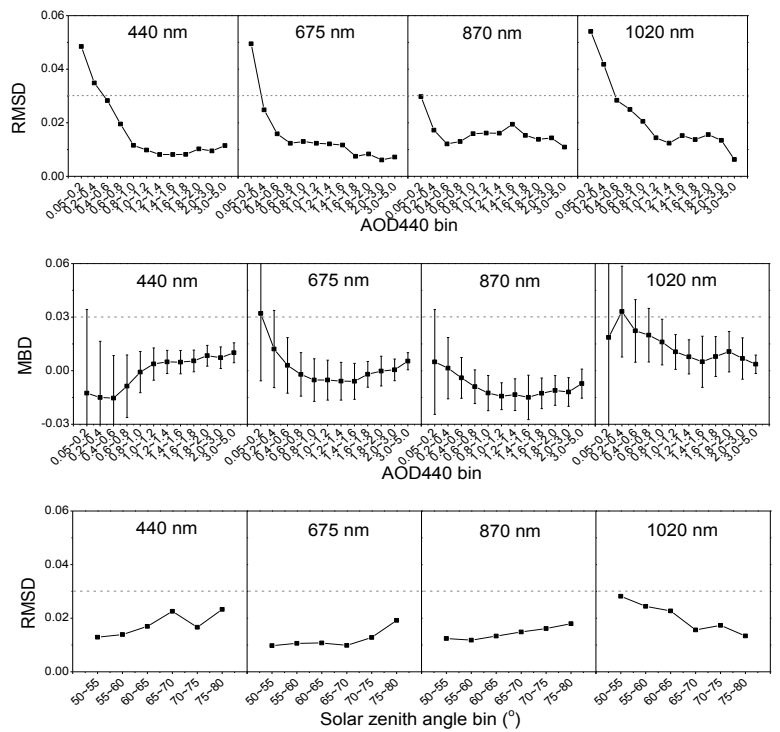


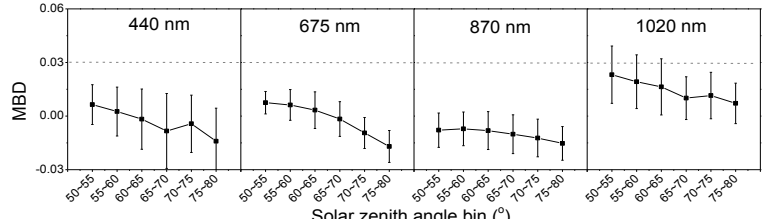

Solar zenith angle bin $\left(^{\circ}\right)$
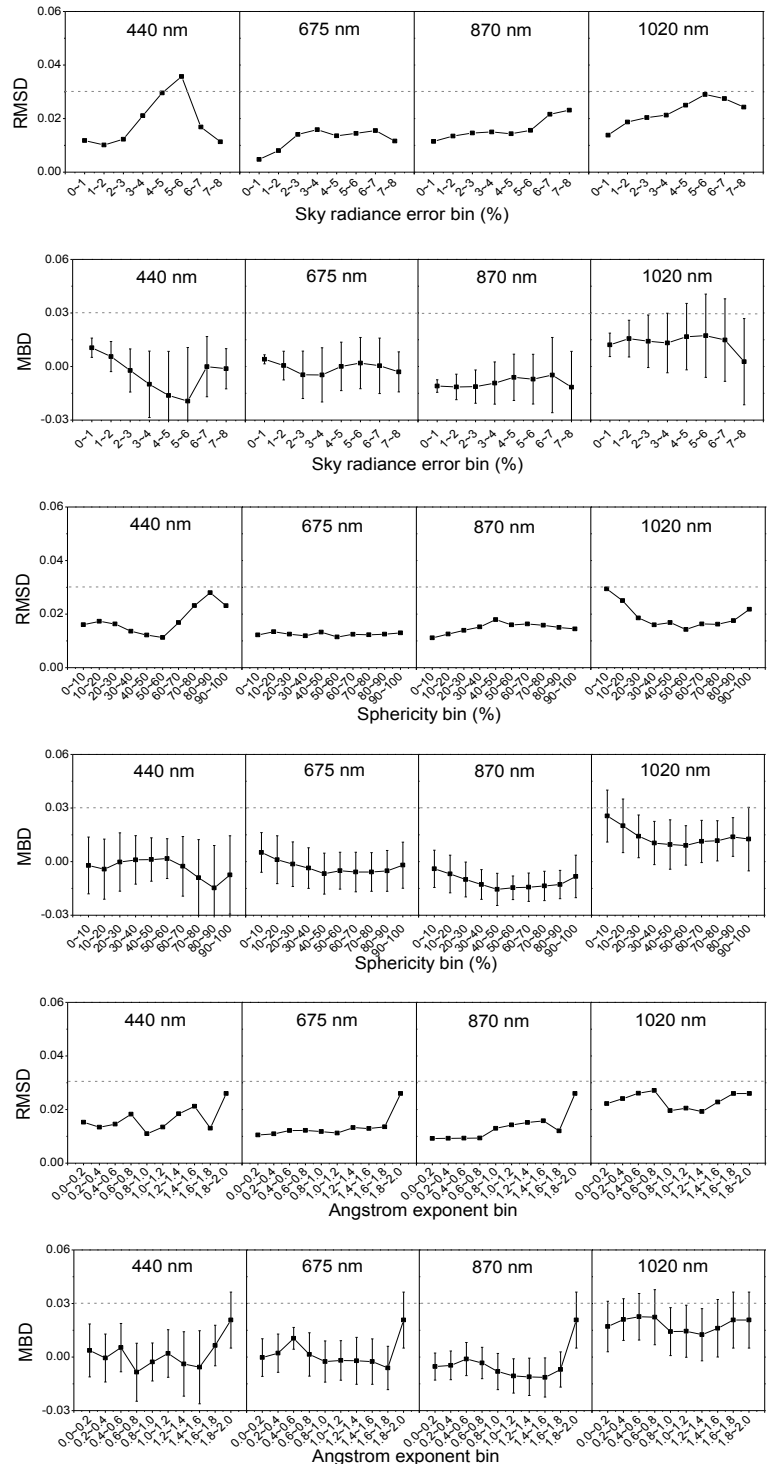

Figure 2 SSAs deviations (RMSD, MBD and STD) of DVL and AERONET as a function of AOD (440 nm), SZ, Sky error, Sphericity and Ángström exponent, at 4 wavelengths of 440, 675,870 and $1020 \mathrm{~nm}$, respectively.

\section{3 g comparison}

Figure 2 shows the RMSD and MBD deviations of the SSAs retrieved by DVL and AERONET, as a function of AOD (440 $\mathrm{nm}), \mathrm{SZ}$, Sky error, Sphericity and Ángström exponent, at 4 wavelengths of $440,675,870$ and $1020 \mathrm{~nm}$, respectively.

a) Change with AOD (440 nm)

The $g$ deviations (RMSD, MBD and STD) of DVL and AERONET decrease the AOD $(440 \mathrm{~nm})$ for the 4 wavelengths. When AOD $(440 \mathrm{~nm})>0.4$, the deviations tend to be stable, with RMSD values of about 0.01 and the absolute value of MBD of about 0.05 at 675,870 and $1020 \mathrm{~nm}$. Although relatively higher deviation presents at $440 \mathrm{~nm}$, the RMSD and absolute value of MBD are less than 0.04 at all the AOD $(440 \mathrm{~nm})$ intervals.

b) Change with $\mathrm{SZ}$

The $g$ deviations (RMSD, MBD and STD) show a downward trend with the solar zenith angle, especially for the 675,870 and $1020 \mathrm{~nm}$ wavelengths. When the SZ increased from $50^{\circ}$ to $80^{\circ}$, the RMSDs decrease from 0.02 to 0.01 and the absolute value of MBD decrease from 0.015 to 0 . This can be explained by the fact that, the coverage of the scattering angle range is an important factor affecting aerosol information content and aerosol $\mathrm{g}$ retrievals, especially for the DVL method as it relies on the integral over the entire scattering angle region.

c) Change with Sky error

Overall, the g deviations (RMSD, MBD and STD) increase with the Sky residual error. The trend is obvious for the $440 \mathrm{~nm}$ wavelength, while the RMSD and MBD of the other 3 wavelengths increase slightly with sky error. However, the STD of all 4 wavelength increase significantly with the sky error. That is because that sky residual error is very sensitive to the instrumental measurements and forward model errors, especially the random errors. It is usually used to indicate the quality of inversion.

d) Change with spherical particles proportion and Ángström exponent

The g deviations (RMSD, MBD and STD) do not change significantly with both the particles' spherical proportion and Ángström exponent. This indicate that the DVL algorithm retrievals of $\mathrm{g}$ are not sensitive to the particles shape and size.
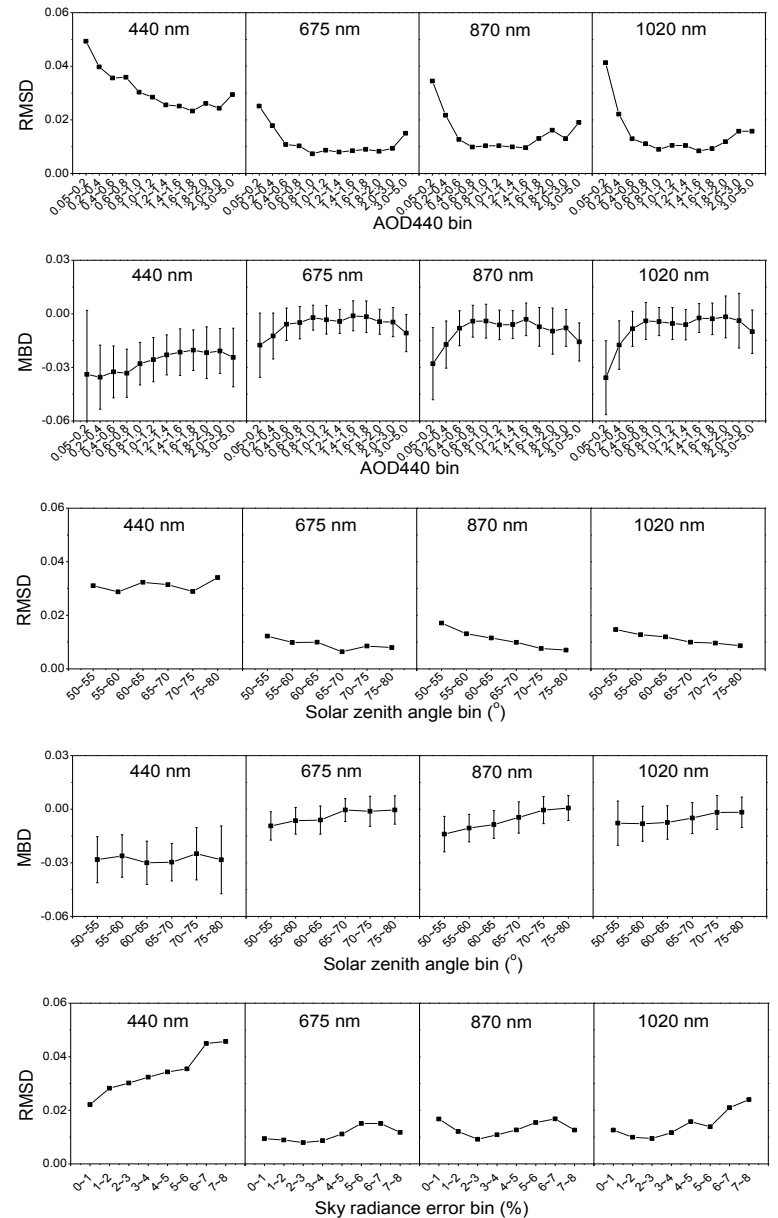


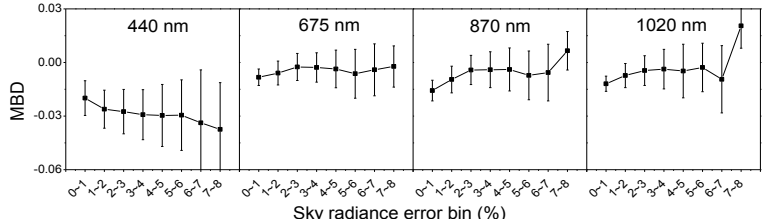

Sky radiance error bin $(\%)$

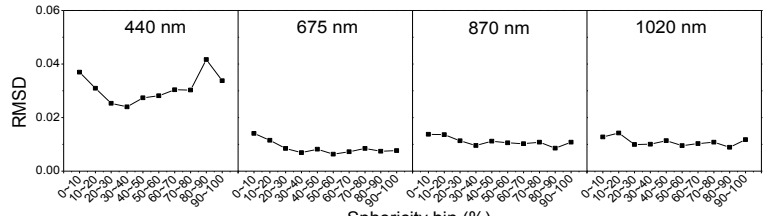

Sphericity bin (\%)
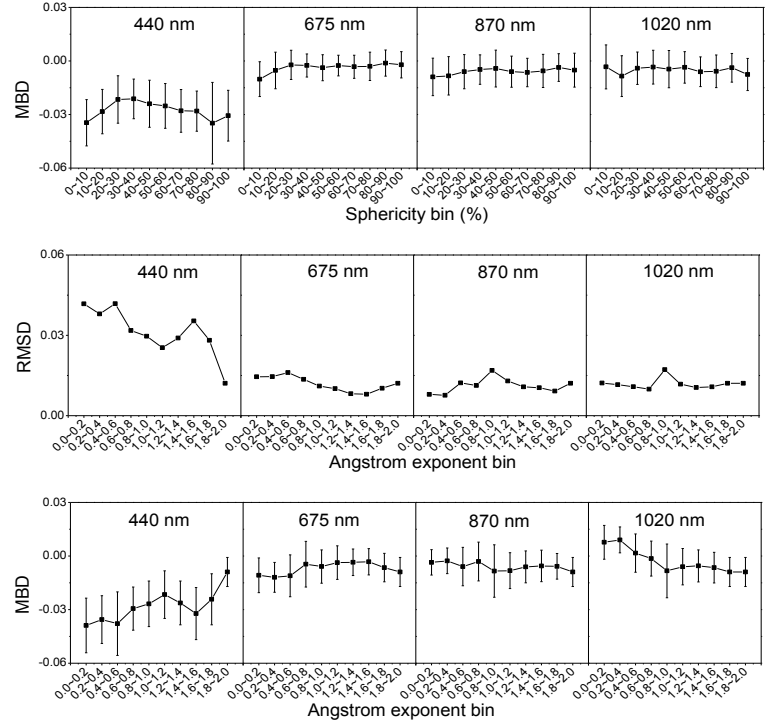

Figure 3 Aerosol asymmetry factor g deviations (RMSD, MBD and STD) of DVL and AERONET as a function of AOD (440 $\mathrm{nm}), \mathrm{SZ}$, Sky error, Sphericity and Ángström exponent, at 4 wavelengths of $440,675,870$ and $1020 \mathrm{~nm}$, respectively.

\section{CONCLUSION}

The DVL algorithm was applied to the AERONET observations, and the aerosol SSA and $g$ are retrieved at 4 wavelengths (440, 675,870 and $1020 \mathrm{~nm}$ ) and the results are compared with AERONET version 2 level 2.0 official products. The SSA and g deviations (RMSD, MBD and STD) of DVL and AERONET algorithm are evaluated considering five factors that probably affecting the retrievals quality.

The comparison shows that both the DVL SSA and $g$ were well correlated with those of AERONET. The SSA RMSD and MBD deviations between them are 0.025 and \pm 0.015 respectively, below the AERONET declared SSA uncertainty of 0.03 for all wavelengths. The $g$ deviations are rather small for the 675,870 and $1020 \mathrm{~nm}$ bands, with RMSD about 0.01 and MBD smaller than 0.006. However, DVL underestimated g about 0.03 at 440 $\mathrm{nm}$ band compared to AERONET. Both the SSA and $g$ deviations were found decrease with the AOD and the solar zenith angle, and increase with sky residual error. However, the deviations do not exhibited distinctly trends for to the sphericity proportion and Ángström exponent, indicating that the algorithm has comparative suitability for non-spherical large particles with other spherical aerosol particles.

The DVL results are suitable for the evaluation of aerosol direct radiative effects of different aerosol types.

\section{ACKNOWLEDGEMENTS}

This work is supported by the National Natural Science Foundation of China (41601385) and the National Key R\&D Program of China (2016YFE0201400). We thank the PIs Hongbin Chen and Philippe Goloub for their effort in maintaining the Beijing AERONET site used in this investigation.

\section{REFERENCES}

Devaux, C., Vermeulen, A., Deuzé, J. L., Dubuisson, P., Herman, M., Santer, R. and Verbrugghe, M., 1998. Retrieval of aerosol single-scattering albedo from ground-based measurements: Application to observational data, Journal of Geophysical Research: Atmospheres 103, pp. 8753-8761.

Dubovik, O. and King, M. D., 2000. A flexible inversion algorithm for retrieval of aerosol optical properties from Sun and sky radiance measurements. Journal of Geophysical Research: Atmospheres 105, pp. 20673-20696.

Dubovik, O., Smirnov, A., Holben, B. N., King, M. D., Kaufman, Y. J., Eck, T. F. and Slutsker, I., 2000. Accuracy assessments of aerosol optical properties retrieved from Aerosol Robotic Network (AERONET) Sun and sky radiance measurements, Journal of Geophysical Research: Atmospheres 105, pp. 9791-9806.

Hansen, J., Sato, M., and Ruedy, R., 1997. Radiative forcing and climate response. Journal of Geophysical Research: Atmospheres 102, pp. 6831-6864.

Holben, B. N. (2002): AERONETcriteria_final1.pdf. https://aeronet.gsfc.nasa.gov/new_web/publications.html.

Holben, B. N., Eck, T., Slutsker, I., Tanre, D., Buis, J., Setzer, A., Vermote, E., Reagan, J., Kaufman, Y., and Nakajima, T., 1998. AERONET - A federated instrument network and data archive for aerosol characterization. Remote sensing of environment 66, pp. 1-16.

Li, Z., Goloub, P., Devaux, C., Gu, X., Deuzé, J. L., Qiao, Y. and Zhao, F., 2006. Retrieval of aerosol optical and physical properties from ground-based spectral, multi-angular, and polarized sun-photometer measurements. Remote Sensing of Environment 101, pp. 519-533.

Li, Z., Li, D., Li, K., Xu, H., Chen, X., Chen, C., Xie, Y., L. Li, $\mathrm{Li}, \mathrm{L}$., and Li, W., 2015. Sun-sky radiometer observation network with the extension of multi-wavelength polarization measurements. J. Remote Sens 19, pp. 495-519.

Nakajima, T., Tonna, G., Rao, R., Boi, P., Kaufman, Y. and Holben, B. N., 1996. Use of sky brightness measurements from ground for remote sensing of particulate polydispersions. Applied Optics 35, pp. 2672-2686.

Vermeulen, A., Devaux, C., and Herman, M., 2000. Retrieval of the scattering and microphysical properties of aerosols from ground-based optical measurements including polarization. I. Method. Applied optics 39, pp. 6207-6220.

Wang, M. and Gordon, H. R., 1993. Retrieval of the columnar aerosol phase function and single-scattering albedo from sky radiance over the ocean: simulations. Applied Optics 32, pp. 4598-4609. 
The International Archives of the Photogrammetry, Remote Sensing and Spatial Information Sciences, Volume XLII-3, 2018 ISPRS TC III Mid-term Symposium "Developments, Technologies and Applications in Remote Sensing", 7-10 May, Beijing, China

Yu, H., Kaufman, Y., Chin, M., Feingold, G., Remer, L., Anderson, T., Balkanski, Y., Bellouin, N., Boucher, O. and Christopher, S., 2006. A review of measurement-based assessments of the aerosol direct radiative effect and forcing. Atmospheric Chemistry and Physics 6, pp. 613-666. 